\title{
The Supersymmetry Index and the Construction of Modular Invariants
}

\author{
N.P. Warner* \\ Mathematics Department, Massachusetts Institute of Technology, Cambridge, MA 02139, USA
}

\begin{abstract}
Relationships between the modular properties of affine $G$ characters and the modular properties of the affine characters of regular subgroups of $G$ are derived by considering the branching functions that appear in calculation of the index of the Dirac-Ramond operator on super-coset models. Various applications of these relationships are described, and in particular a simple algorithm is given for generating modular invariant combinations of characters of affine $G$ at any level by using the shift vector method on suitably chosen Lorentzian, self-dual lattices.
\end{abstract}

\section{Introduction}

The construction of modular invariants for arbitrary unitary representations of Kac-Moody algebras has been of interest for some time. Moreover, with the advent of $N=2$ supersymmetric coset models [1] the construction of such invariants has become of some significance for string theory. My intention here is to introduce a technique that helps one to classify these invariants, and as a by-product yields an elementary computational algorithm for generating vast numbers of (and possibly all) modular invariant combinations of characters for Kac-Moody representations of any level.

The basic idea is decribed in Sect. 2 of this paper and rests on a simple, but powerful identity that can be derived for any supersymmetric coset model. This identity was essentially derived in [2], however the emphasis there was on the properties of the coset model, whereas here the coset model in literally orthogonal to the discussion. This identity will be used to study the modular invariants of affine $G$-representations via embeddings of regular subgroups, $H$, of $G$.

In Sect. 3 a number of applications of the results of Sect. 2 will be given. In particular, one can obtain an elementary derivation of the formula for modular

\footnotetext{
* Work supported in part by funds provided by the NSF under grant No. 87-08447 and by the U.S. Department of Energy (D.O.E) under contract \# DE-AC02-76ER0306, and also by a fellowship from the Alfred P. Sloan foundation
} 
transformations of affine $G$-characters. One can also easily recover, and generalize, the work of [3] and [4] in which modular invariants of affine $H$ can be obtained via special conformal embeddings of $H$ into some larger group. Perhaps the most interesting application is that if one knows a modular invariant for some regular subgroup $H$ of some group $G$ then one can generate a modular invariant for $G$. Indeed one can generate all the modular invariants for $G$ in this manner. If one takes $H$ to be the maximal torus of $G$ then the problem of finding modular invariants for affine $G$ (at any level) reduces to finding modular invariants for a set of bosons compactified on a suitably chosen lattice. As a consequence one can obtain new modular invariants for $G$ by merely generating a particular family of Lorentzian, self-dual lattices using the shift vector method [5]. The latter technique is a simple and established piece of lattice technology that has found considerable application in generating modular invariants in string theories based on level one representations of Kac-Moody algebras. Here this technique finds broader application.

As one might expect, there is a price to be paid for generating modular invariants so easily. The problem is that one generates modular invariant combinations of characters that are, in general, not good partition functions for corformal field theories. The modular invariants that one generates do not necessarily have positive coefficients in their $q$-expansions. That is, the "occupation numbers" can be negative. This problem will be discussed in Sect. 4 .

\section{Supersymmetric Cosets and Modular Relations}

Consider the Kac-Moody algebra $\hat{\mathscr{G}}$ that is constructed from some rank $\ell$, semisimple Lie algebra $\mathscr{G}{ }^{1}{ }^{1}$ The Lie group corresponding to $\mathscr{G}$ will be denoted by $G$. A unitary representation of $\hat{\mathscr{G}}$ will be denoted by its level, $k$, and by a dominant weight, $\Lambda$, of the (finite) underlying Lie algebra $\mathscr{G}$. As usual, the label $\Lambda$ is the highest weight of the $G$-representation that forms the ground-state of the KacMoody representation, and $\Lambda$ is required to satisfy the inequality $\Lambda \cdot \theta \leq k$, where $\theta$ is the highest root of $\mathscr{G}$. The co-root lattice (i.e. the lattice generated by the long roots) of $\mathscr{G}$ will be denoted by $\Gamma(\mathscr{G})$. Let $H$ be a subgroup the Lie group $G$. Suppose that $H$ also has rank $\ell$, and is semi-simple except possibly for $U(1)$ factors. Because $H$ has the same rank as $G$, it follows that is must be regularly embedded, that is, the co-root lattice $\Gamma(\mathscr{H})$ of $H$ is a sub-lattice of $\Gamma(\mathscr{G})$.

The super-GKO construction [1] for the coset $G / H$ can be thought of in terms of the usual GKO construction [8] for the coset model $\mathscr{M}=(G \times S O(d)) / H$, where $d$ is the dimension of $G / H$ and $H$ is diagonally embedded into $G$ and $S O(d)$. The $S O(d)$ factor represents the bosonized fermions of the super-GKO model, and the embedding of $H$ into $S O(d)$ is induced via the $H$ action on the tangent space of $G / H$. The embedding index (or level) of $H$ in $S O(d)$ is $g-g_{H}$, where $g$ and $g_{H}$ are the dual Coxeter numbers of $G$ and $H$ respectively. ${ }^{2}$ The

\footnotetext{
1 For a review of the relevant material see [6], or see the appendix of [7]

2 If $G$ and $H$ are products of several factors then $g-g_{H}$ is to be thought of as a vector in the obvious manner. The dual Coxeter number of $U(1)$ factors is defined to be zero
} 
characters of the coset model $\mathscr{M}$ are branching functions [6], $b_{\Lambda, \tilde{A}} \lambda$, defined via:

$$
\chi_{\Lambda}^{(G)} \chi_{\tilde{\Lambda}}^{S O}=b_{\Lambda, \tilde{\Lambda}}^{\lambda} \chi_{\lambda}^{(H)},
$$

where $\chi_{\Lambda}^{(G)}$ is the level $k, \Lambda$ character of affine $G ; \chi_{\tilde{\Lambda}}^{S O}$ is the level $1, \tilde{\Lambda}$ character of affine $S O(d)$ and $\chi_{\lambda}^{(H)}$ is the level $k+g-g_{H}, \lambda$ character of affine $H$. The index $\lambda$ is to be summed over all characters of $\tilde{\mathscr{H}}$ at level $\left(k+g-g_{H}\right)$.

The Ramond sector of the coset model $\mathscr{M}$ is obtained from the spinor representations of affine $S O(d)$. For every $\Lambda$ and $\lambda$ one can define an index branching function, $I_{A}{ }^{\lambda}$,

$$
\chi_{\Lambda}^{(G)}\left(\chi_{+}^{S O}-\chi_{-}^{S O}\right)=I_{\Lambda}^{\lambda} \chi_{\lambda}^{(H)},
$$

where $\chi_{ \pm}^{S O}$ are the characters of the two spinor representations of affine $S O(d)$. (Note that $d$ is always even.) The branching function $I_{A}{ }^{\lambda}$ can be thought of as the contribution of the $(\Lambda, \lambda)$ representation of affine $(G, H)$ to the index of the Dirac-Ramond operator on the super-coset model [2]. Now take the characters to be functions of $v$ and $\tau$, where $v$ is a vector of parameters corresponding to a Cartan subalgebra of $H$. This means that $\chi_{A}^{(G)}$ and $\chi_{ \pm}^{S O}$ are to be viewed as functions of $v$ and $\tau$ as induced by the embedding of $H$. In particular, it means that the chiral character $\left(\chi_{+}^{S O}-\chi_{-}^{S O}\right)$ does not vanish identically. Indeed,

$$
\left(\chi_{+}^{S O}-\chi_{-}^{S O}\right)(v, \tau)=\prod_{\alpha \in t^{+}}\left[\frac{\theta_{1}(\alpha \cdot v \mid \tau)}{\eta(\tau)}\right],
$$

where $t^{+}=\Delta^{+}(\mathscr{G}) \backslash \Delta^{+}(\mathscr{H})$, and $\Delta^{+}(\mathscr{A})$ denotes the positive roots of $\mathscr{A}$. By definition, $I_{A}{ }^{\lambda}$ is independent of $v$, and by the usual index arguments it must also be independent of $\tau$. Thus $I_{\Lambda}{ }^{\lambda}$ is a constant.

Consider the general modular transformation

$$
\tau \rightarrow \frac{a \tau+b}{c \tau+d}, \quad v \rightarrow \frac{v}{c \tau+d}
$$

acting on both sides of Eq. (2.2). First, there are overall phases, usually known as modular anomalies, that have the form $\exp \left[i \pi \alpha v^{2} /(c \tau+d)\right]$, where $\alpha$ is a constant. These anomalous phases are the same on both sides of the transformed Eq. (2.2), and thus cancel. Therefore the modular anomaly terms will be neglected henceforth. The affine $G$ and $H$ characters transform according to

$$
\chi_{\Lambda}^{(G)} \rightarrow \mathscr{U}_{\Lambda}^{A^{\prime}} \chi_{\Lambda^{\prime}}^{(G)} ; \quad \chi_{\lambda}^{(H)} \rightarrow \mathscr{V}_{\lambda}^{\lambda^{\prime}} \chi_{\lambda^{\prime}}^{(H)},
$$

where $\mathscr{U}_{A}{ }^{\Lambda^{\prime}}$ and $\mathscr{V}_{\lambda}{ }^{\lambda^{\prime}}$ are unitary matrices [6]. Moreover the chiral character of $S O(d)$, given in (2.3), is a modular invariant up to twelfth roots of unity. More precisely, under $\tau \rightarrow \tau+1,\left(\chi_{+}^{S O}-\chi_{-}^{S O}\right)$ gets multiplied by a factor of $e^{i \pi\left|t^{+}\right| / 6}$, where $\left|t^{+}\right|$is the number of positive roots in $t^{+}$. Under $\tau \rightarrow-1 / \tau$ the factor is simply $(-i)^{\left|t^{+}\right|}$. It therefore follows from Eq. (2.2) that, under the modular transformation (2.4), one has: $I_{\Lambda}^{\lambda} \rightarrow e^{i \phi} \mathscr{U}_{\Lambda} \Lambda^{\Lambda^{\prime}} I_{\Lambda^{\prime}}{ }^{\lambda^{\prime}} \mathscr{V}_{\lambda^{\prime}}^{* \lambda}$, where $e^{i \phi}$ is a twelfth root of unity. However, as was noted above, the index branching functions are constants and hence are trivially invariant under modular transformations. It follows that for any modular transformation:

$$
\mathscr{U}_{\Lambda}^{\Lambda^{\prime}} I_{\Lambda^{\prime}}{ }^{\lambda}=e^{i \phi} I_{\lambda}^{\lambda^{\prime}} \mathscr{V}_{\lambda^{\prime}}{ }^{\lambda},
$$


where $\phi=-\frac{\pi}{6}\left|t^{+}\right|$for $\tau \rightarrow \tau+1$ and $\phi=\frac{\pi}{2}\left|t^{+}\right|$for $\tau \rightarrow-1 / \tau$. This somewhat remarkable identity yields non-trivial relations between the modular properties of level $k$ characters of $\hat{\mathscr{G}}$, and level $k+g-g_{H}$ characters of $\hat{\mathscr{H}}$. However, to use this identity one must first determine $I_{A}{ }^{\lambda}$.

It is a virtually trivial application of the Weyl-Kac character formula to derive an explicit formula for $I_{\Lambda}^{\lambda}$ [2]: Indeed, multiplying the character formula for $\chi_{\Lambda}^{(G)}$ by (2.3) one finds that the result instantly decomposes into characters $\chi_{\lambda}^{(H)}$.

Let $W(G)$ and $W(H)$ denote the (finite) Weyl groups of $G$ and $H$ respectively, and let $p$ be the number defined by

$$
p=\left|\frac{W(G)}{W(H)}\right|\left|\frac{\Gamma(\mathscr{G})}{\Gamma(\mathscr{H})}\right| .
$$

Consider all the vectors of the form:

$$
v=w\left(\Lambda+\varrho_{G}\right)+(k+g) \mu,
$$

where $w \in W(G)$ and $\mu \in \Gamma(\mathscr{G})$. Then [2] there are precisely $p$ vectors $v$ of the form (2.8) that satisfy the following conditions:

$$
\left(v-\varrho_{H}\right) \cdot \alpha \geq 0
$$

for all positive roots, $\alpha$, of $\mathscr{H}$, and

$$
\left(v-\varrho_{H}\right) \cdot \psi \leq\left(k+g-g_{H}\right),
$$

where $\psi$ is the highest root of $H$. In other words there are precisely $p$ vectors of the form $v$ such that $\lambda=v-\varrho_{H}$ is an admissible representation label for $\hat{\mathscr{H}}$ at level $k+g-g_{H}$. For a given $\Lambda$, let $\mathscr{R}(\Lambda)$ denote the set of all these admissible labels of the form $\lambda=v-\varrho_{H}$. One then finds [2] that $I_{\Lambda}{ }^{\lambda}$ vanishes unless $\lambda$ is an element of $\mathscr{R}(\Lambda)$, in which case one has

$$
I_{\Lambda}^{\lambda}=\varepsilon(w)
$$

where $\varepsilon(w)$ is the sign of the determinant of the Weyl reflection, $w \in W(G)$, in Eq. (2.8).

There is a simple algorithm for computing $\mathscr{R}(\Lambda)$. Take coset representatives, $w$, of $W(G) / W(H)$ and representative vectors $\mu$ of $\Gamma(\mathscr{G}) / \Gamma(\mathscr{H})$. Consider the corresponding vectors $v$ defined in Eq. (2.8). For each such $v$ pick the representative $\mu$ so that $v$ has the minimum possible length. That is, if necessary, modify $v$ by adding to it a vector of the form $(k+g) \mu_{H}, \mu_{H} \in \Gamma(\mathscr{H})$ so that the result has minimal length. Having chosen such a minimal $v$, act with a Weyl rotation of $W(H)$ so as to place $v$ in the fundamental Weyl chamber of $H$, i.e. so as to ensure that $v \cdot \alpha \geq 0$ for all positive roots $\alpha$ of $\mathscr{H}$. One will find that the rotated form of $v$ in fact obeys $v \cdot \alpha>0$ for all positive roots of $H$, and thus $v-\varrho_{H}$ satisfies (2.9). Moreover, the fact that one has minimized the length of $v$ guarantees that $(2.10)$ is satisfied. It is also elementary to see that all the vectors $\lambda=v-\varrho_{H}$ generated in this manner are distinct. From this it also follows that

$$
\sum_{\lambda} I_{\Lambda}^{\lambda} I_{\Lambda^{\prime}}^{\lambda}=p \delta_{\Lambda \Lambda^{\prime}}
$$

where $p$ is given by Eq. (2.7). 


\section{Applications}

Observe that if $M^{\Lambda \Lambda^{\prime}} \chi_{\Lambda}^{(G)} \overline{\left(\chi_{\Lambda^{\prime}}^{G}\right)}$ is a modular invariant for $\hat{\mathscr{G}}$ at level $k$, then $P^{\lambda \lambda^{\prime}} \chi_{\lambda}^{(H)} \overline{\left(\chi_{\lambda^{\prime}}^{(H)}\right)}$ is modular invariant for $\hat{\mathscr{H}}$ at level $k+g-g_{H}$, where

$$
P^{\lambda \lambda^{\prime}} \equiv M^{\Lambda \Lambda^{\prime}} I_{\Lambda}{ }^{\lambda} I_{\Lambda^{\prime}}{ }^{\lambda^{\prime}}
$$

Conversely given an invariant, $P^{\lambda \lambda^{\prime}}$, of $\hat{\mathscr{H}}$ one can obtain one for $\hat{\mathscr{G}}$ by taking

$$
M^{\Lambda \Lambda^{\prime}}=P^{\lambda \lambda^{\prime}} I_{\Lambda}^{\lambda} I_{\Lambda^{\prime}}^{\lambda} .
$$

More generally, if $\mathscr{H}$ is a product: $\mathscr{H}=\mathscr{H}_{1} \times \mathscr{H}_{2} \times \cdots \times \mathscr{H}_{n}$, then given modular invariants for $\hat{\mathscr{G}}$ and $\hat{\mathscr{H}}_{2}, \ldots, \hat{\mathscr{H}}_{n}$, one can obtain an invariant for $\hat{\mathscr{H}}_{1}$ by taking the tensor product of $I$ with itself and contracting the indices corresponding to $\hat{\mathscr{G}}$ and $\hat{\mathscr{H}}_{2}, \ldots, \hat{\mathscr{H}}_{n}$ with the appropriate given modular invariant.

The latter procedure has essentially been applied in a very restricted context in [3] and [4]. Specifically, one can take $k=0$ and $\Lambda=0$ (i.e. the trivial, one dimensional representation for $\hat{\mathscr{G}}$ ). Suppose that one then takes the diagonal invariants for $\hat{\mathscr{H}}_{2}, \ldots, \hat{\mathscr{H}}_{n}$, then one obtains an invariant for $\hat{\mathscr{H}}_{1}$. In [3] $G$ was taken to be an $A, D$ or $E$ Lie group and $H$ was $S U(2) \times H_{2}$ with an embedding into $G$ in which the $S U(2)$ was generated by $E_{\theta}, E_{-\theta}$ and $\theta^{i} X_{i}$, where $\theta$ is the highest root of $\mathscr{G}$ and $X_{i}$ denotes the Cartan subalgebra. The corresponding $S U(2)$ embedding into $S O(d)$ has level $g-2$, and the induced modular invariant for $S U(2)$ was found to be a simple linear combination of the $A_{g-2}$ invariant and the type $\mathscr{G}$ invariant. Thus this technique afforded one some link between A-D-E modular invariants of $S U(2)$ and the corresponding A-D-E Lie group.

The arguments of [3] and [4] rely on gauging the group $\mathrm{H}_{2}$ and then sending the coupling constant to infinity in order to induce the new modular invariant for $\mathscr{H}_{1}$. This technique is only valid for conformal embeddings of $H$. In other words, if one uses the gauging trick to remove the $\mathscr{H}_{2}$ Hilbert space then one has only generated a modular invariant for the orthogonal Hilbert space, which is only a finitely reducible representation of $\mathscr{H}_{1}$ for conformal embeddings. The advantage of the procedure outlined above is that it does not require one to take $k=0$ and $\Lambda=0$, or take a conformal embedding. The Hilbert space orthogonal to that of $\mathscr{H}_{2} \times \cdots \times \mathscr{H}_{n}$ is essentially a product of a coset model and representations of $\hat{\mathscr{H}}_{1}$. The modular invariant for the coset has been chosen to be the trivial (constant) index branching function, and we are then left with an invariant for $\mathscr{H}_{1}$.

Another elementary application of (2.6) is to derive the general formula for the modular transformation of $\hat{\mathscr{G}}$ characters. In particular consider the transformation $\tau \rightarrow-1 / \tau$. Take $H=(U(1))^{\ell}$, where $\ell$ is the $\operatorname{rank}$ of $G$, and set $\Gamma(\mathscr{H})=\Gamma(\mathscr{G})$. Thus the $H$-theory is that of $\ell$ free bosons compactified on the integral lattice $\sqrt{k+g} \Gamma(\mathscr{G})$. The $H$-partition functions are then the shifted theta functions of this lattice, divided by $[\eta(\tau)]^{\ell}$ :

$$
\chi_{\lambda}^{(H)}(\tau)=[\eta(\tau)]^{-\ell} \sum_{v \in \Gamma(\mathscr{G})} \exp \left[i \pi(k+g)\left(v+\frac{\lambda}{(k+g)}\right)^{2} \tau\right] .
$$


By Poisson resummation one knows that

$$
\chi_{\lambda}^{(H)}(-1 / \tau)=\left|\frac{\Gamma^{*}}{(k+g) \Gamma}\right|^{-\frac{1}{2}} \sum_{\mu} e^{-2 \pi i \frac{\mu \cdot \lambda}{(k+g)}} \chi_{\lambda}^{(H)}(\tau)
$$

where $\Gamma^{*}$ is the dual of $\Gamma \equiv \Gamma(\mathscr{G})$ and the sum is taken over $\mu \in \frac{\Gamma^{*}}{(k+g) \Gamma}$.
Using the results given at the end of the last section one sees that:

$$
I_{\Lambda}^{\lambda}= \begin{cases}\varepsilon(w) & \text { if } \lambda=w\left(\Lambda+\varrho_{G}\right) \\ 0 & \text { otherwise }\end{cases}
$$

where $w \in W(G)$. In Eq. (2.6) take $\lambda=\Lambda^{\prime}+\varrho_{G}$, for some fixed $\Lambda^{\prime}$, then using (3.4) and (3.5) one obtains the following matrix for the transformation of affine $G$-characters under $\tau \rightarrow-1 / \tau$ :

$$
S_{\Lambda}^{\Lambda^{\prime}}=(i)^{\left|\Delta^{+}\right|}\left|\frac{\Gamma^{*}}{(k+g) \Gamma}\right|^{-\frac{1}{2}} \sum_{w \in W(G)} \varepsilon(w) \exp \left[-\frac{2 \pi i}{(k+g)} w\left(\Lambda+\varrho_{G}\right) \cdot\left(\Lambda^{\prime}+\varrho_{G}\right)\right]
$$

where $\left|\Delta^{+}\right|$is the number of positive roots of $G$. This result is well known (see Chap. 13 of [6]), but the proof given here $I$ believe to be somewhat simpler than the usual one.

Perhaps the most novel application of Eq. (2.6) is the process by which one can generate new modular invariants for $\hat{\mathscr{G}}$ given modular invariants for $\hat{\mathscr{H}}$. There are a priori a myriad of ways in which one can do this. For example, given that one knows all the modular invariants for $S U(2)$ [9], one can embed products of $S U(2)$ into $G$, adding $U(1)$ factors if necessary, then choose $A, D$, or $E$ invariants for each of the $S U(2)$ factors and, for instance, choose the trivial modular invariant for each of the $U(1)$ factors. From each such choice one obtains a modular invariant for $G$. The simplest non-trivial example of this procedure of using $S U(2)$ factors is to take $\mathscr{G}=S U(3)$ at level $k$, and take $H=S U(2) \times U(1)$, where $S U(2)$ has level $(k+1)$. For the present $I$ will always use the trivial invariant for the $U(1)$ factors. One would naturally expect some $S U$ (3) analogue of the type $D$ modular invariants for odd values of $k$, and some analogue of the type $E$ modular invariants when $k$ is 9,15 or 27 . For example, taking $k=3$ and using the $D_{4}$ modular invariant of $S U(2)$, one obtains an $S U_{3}(3)$ invariant of the form $3 A-I_{3}$, where $A$ is the trivial (diagonal) modular invariant and

$$
I_{3}=\left|\chi_{0,0}+\chi_{3,0}+\chi_{0,3}\right|^{2}+3\left|\chi_{1,1}\right|^{2}
$$

where the subscripts on the $\chi$ 's are the Dynkin labels of the highest weight vectors, $A$. Note that $S U(3) \hookrightarrow S O_{1}(8)$ is a conformal embedding and one can check that (3.7) is in fact the partition function of the Englert-Neveu lattice [10] based upon $S O(8)$. The $S U(3)$ analogues of the type $D$ modular invariants are readily computed for higher values of $k$, and, for example, for $k=5$ and $k=7$ 
one finds invariants of the form $3 A-I_{k}$, where

$$
\begin{aligned}
I_{5}= & \left|\chi_{0,0}-\chi_{2,2}\right|^{2}+4\left|\chi_{1,1}\right|^{2}+\left|\chi_{1,4}+\chi_{4,1}\right|^{2} \\
& +\left\{\left(\left|\chi_{5,0}-\chi_{1,2}\right|^{2}+\left|\chi_{4,0}+\chi_{1,0}\right|^{2}+4\left|\chi_{3,1}\right|^{2}\right)+\left(n_{1} \leftrightarrow n_{2}\right)\right\}, \\
I_{7}= & \left|\chi_{0,0}+\chi_{5,2}+\chi_{2,5}\right|^{2}+\left|\chi_{0,6}+\chi_{6,0}-\chi_{2,2}\right|^{2} \\
& +\left\{\left(\left|\chi_{7,0}+\chi_{0,2}+\chi_{0,5}\right|^{2}+\left|\chi_{6,1}+\chi_{0,1}-\chi_{2,3}\right|^{2}\right)+\left(n_{1} \leftrightarrow n_{2}\right)\right\},
\end{aligned}
$$

and where $\left(n_{1} \leftrightarrow n_{2}\right)$ indicates an identical expression to the preceeding one in parentheses, except that the Dynkin labels $n_{1}$ and $n_{2}$ are to be interchanged. One can also easily compute the $S U(3)$ analogues of the other $D$ invariants or for the $E$ invariants.

At this point one should observe that there is a problem with some of the modular invariants that have been generated thus far. While they are indeed modular invariant, they are not necessarily good partition functions for a conformal field theory. This is because some of the occupation numbers might be negative since some of the characters appear in the invariants with negative coefficients. This difficulty will be discussed later since I first wish to describe a more fundamental and systematic method of generating modular invariants for $\hat{\mathscr{G}}$.

It is important to note that given a modular invariant $\tilde{M}^{\Lambda \Lambda^{\prime}}$ of $\hat{\mathscr{G}}$ at level $k$, if one uses (3.1) to obtain a modular invariant $P^{\lambda \lambda^{\prime}}$ for $\hat{\mathscr{H}}$ and then uses (3.2) to obtain a modular invariant $M^{\Lambda \Lambda^{\prime}}$ for $\hat{\mathscr{G}}$ again, it follows from (2.12) that

$$
M^{\Lambda \Lambda^{\prime}}=p \tilde{M}^{\Lambda \Lambda^{\prime}} \text {. }
$$

Therefore every modular invariant of $\hat{\mathscr{G}}$ can be obtained from a modular invariant of $\hat{\mathscr{H}}$. In practice, one also finds that several different invariants of $\hat{\mathscr{H}}$ can lead to the same invariant for $\hat{\mathscr{G}}$.

Consider, once again the situation when $H$ is $(U(1))^{\ell}$, where $\ell$ is the rank of $G$. There is, a priori some ambiguity as to what lattice to choose for $\Gamma(\mathscr{H})$. The obvious choice, and the one that I shall make is to use $\Gamma(\mathscr{G})$, or more precisely $\sqrt{k+g} \Gamma(\mathscr{G})$. Since all modular invariants of $\hat{\mathscr{G}}$ will yield modular invariants for $H=(U(1))^{\ell}$ compactified on this particular lattice, it follows from the considerations above that this lattice suffices to generate all modular invariants of $G$ at level $k$.

The difficulty is that one does not know how to generate all the modular invariants of bosons compactified on a given lattice. However, I believe that it can be done by the algorithm given below, and even if this belief should prove unfounded then at the very least one can generate many, many modular invariants for $G$.

Consider the following Lorentzian self-dual lattice of dimension $(\ell, \ell)$ :

$$
L=\left\{\left(v_{+}, v_{-}\right): v_{+}, v_{-} \in \frac{1}{\sqrt{k+g}} \Gamma^{*}(\mathscr{G}),\left(v_{+}-v_{-}\right) \in \sqrt{k+g} \Gamma(\mathscr{G})\right\}
$$

and consider its partition function

$$
P_{L}(\tau, \bar{\tau})=\sum_{\left(v_{+}, v_{-}\right) \in L} q^{\frac{1}{2} v_{+}^{2}} \bar{q}^{\frac{1}{2} v_{-}^{2}} .
$$


This function is the trivial, or diagonal, modular invariant for $H$. One can easily check that this induces, via (3.2), $|W(G)|$ times the diagonal modular invariant for $G$, i.e. $|W(G)| \sum_{A}\left|\chi_{A}^{(G)}\right|^{2}$.

One can generate new modular invariants for $\mathscr{H}$, and hence for $\mathscr{G}$, by finding a new Lorentzian, self-dual lattice, $L^{\prime}$, that contains $\sqrt{k+g}(\Gamma(\mathscr{G}), \Gamma(\mathscr{G}))$ as a sub-lattice. All such lattices can be generated by the shift vector method [5]. That is, one finds an $(\ell, \ell)$-dimensional vector, $\sigma=\left(\sigma_{+}, \sigma_{-}\right)$, lying on $\frac{1}{\sqrt{k+g}}\left(\Gamma^{*}(\mathscr{G}), \Gamma^{*}(\mathscr{G})\right)$, such that $\sigma \notin L$ but $N \sigma \in L$ for some $N \in \mathbb{Z}, N>1$. Let $N$ be the least such $N$. The vector $\sigma$ must satisfy a level matching condition [11], which can be reduced, without loss of generality, to assuming that $\sigma^{2}=\sigma_{+}{ }^{2}-\sigma_{-}{ }^{2}$ is an even integer [5]. One then defines a new lattice

$$
L^{\prime}=\bigcup_{j=0}^{N-1}\left(L_{0}+j \sigma\right),
$$

where

$$
L_{0}=\{v \in L: v \cdot \sigma \in \mathbb{Z}\} .
$$

The lattice $L^{\prime}$ is even, Lorentzian and self-dual, and so defines a new modular invariant partition function for $\mathscr{H}$. To obtain all the Lorentzian, self-dual lattices than contain $\sqrt{k+g}(\Gamma(\mathscr{G}), \Gamma(\mathscr{G})$ ) as a sub-lattice it may be necessary not only to act with all admissible shift-vectors on $L$, but also to iterate the foregoing procedure on each new lattice that one generates.

As far as the induced modular invariant for $\mathscr{G}$ is concerned, the relevant vectors of $L^{\prime}$ are those of the form

$$
\frac{1}{\sqrt{k+g}}\left(w_{+}\left(\Lambda_{+}+\varrho_{G}\right), w_{-}\left(\Lambda_{-}+\varrho_{G}\right)\right)
$$

where $w_{+}, w_{-} \in W(G)$ and $\Lambda_{+}$and $\Lambda_{-}$are admissible weight labels for some level $k$ representation of $\hat{\mathscr{G}}$. Thus it suffices to restrict attention to shift vectors, or combinations of shift vectors that have this form. The requirement that such vectors have even Lorentzian length is the same as requiring that

$$
\frac{1}{k+g}\left[C_{2}\left(\Lambda_{+}\right)-C_{2}\left(\Lambda_{-}\right)\right] \in \mathbb{Z}
$$

where $C_{2}(\Lambda)$ is the quadratic Casimir of the representation corresponding to $\Lambda$. In other words it suffices to consider the shift vectors, $\sigma$, that can be generated by taking Weyl rotations of the weight labels of all possible primary fields of the level $k, \hat{G}$ theory such that the spin (the difference of left and right moving conformal dimensions) of the primary field is integral. The labels $\left(\Lambda_{+}, \Lambda_{-}\right)$of such fields will then necessarily satisfy Eq. (3.16).

Thus every operator of integral spin can be used to generate new modular invariants for $\hat{\mathscr{G}}$ in an elementary manner. It is also clear that there are only finitely many Lorentzian self dual lattices containing $\sqrt{k+g}(\Gamma(\mathscr{G}), \Gamma(\mathscr{G}))$ and all of them can be enumerated in this manner by applying successive shifts.

As mentioned earlier, the problem with this algorithm is that it not only yields modular invariant partition functions of conformal theories, but it also generates 
a host of modular invariants that cannot be thought of as partition functions. However, if one can classify all the modular invariants of bosons compactified on the lattice $\sqrt{k+g} \Gamma(\mathscr{G})$, then one can classify all modular invariants of $\hat{\mathscr{G}}$ at level $k$. Moreover, it is just possible that the self-dual lattice method will enumerate all such invariants.

As an example of this procedure it is instructive to consider the level $k S U(2)$ invariants. In this instance one has

$$
I_{\ell}^{\ell^{\prime}}=\left(\delta_{(\ell+1)}^{\ell^{\prime}}-\left(\delta_{-(\ell+1)}^{\ell^{\prime}}\right),\right.
$$

where $\ell=0, \ldots, k$ is the usual $S U$ (2) label $(\ell$ is twice the spin of the ground state representation) and $\ell^{\prime}$ is the $U(1)$ charge of a boson $\sqrt{2(k+2)} \partial \phi(z)$ compactified on a lattice with charge spacing $2(k+2)$. The trivial $U(1)$ invariant (3.11) yields the type A invariant (multiplied by $|W(S U(2))|=2$ ). If $k$ is a multiple of 4 one can choose $\sigma=(1, k+1)$ and if $k$ is even but not a multiple of 4 one can take $\sigma=(0, k+2)$. The $S U_{k}(2)$ modular invariant resulting from this is precisely $2(A-D)$, where $A$ and $D$ denote that $A$ and $D$ modular invariants of $S U(2)$ for $k$ even. Alternatively, for any even $k$ one could take $\sigma=\left(\frac{1}{2}(k+2),-\frac{1}{2}(k+2)\right)$ and one gets $2 A-4 D$ or $2(A-D)$ for $\frac{1}{2} k$ even or odd respectively.

If $k$ is 10 or 16 one can generate the invariant $2(D-E)$ by shifts of $(1,7)$ and $(3,15)$ respectively. If $k=28$ one needs two shift vectors, for example the sum of the two modular invariants generated by $(1,11)$ and $(1,19)$ generate the modular invariant $2(E-D)$. These are, of course, not the only shift vectors that generate these modular invariants. One can also generate other combinations of these modular invariants by employing successive shifts. It is thus clear that one can trivially generate all the modular invariants of $S U(2)$ in this manner. Indeed in obtaining all the modular invariant partition functions for $S U_{k}(2)$ the authors of [9] employed a method that is essentially equivalent to the foregoing one.

As a final note on this $S U(2)$ example, there is an amusing and perhaps significant numerological coincidence. With a minor exception for the $E_{7}$ invariant, one can choose the shift vectors required to make the $A, D$ and $E S U$ (2) invariants in such a way that their orders relative to $\sqrt{k+2} \Gamma(S U(2))$ is the same as that of the abelian subgroups of the finite subgroups of $S U(2)$. This is reminiscent of the construction of $c=1$ models by orbifoldizing $S U$ (2) (at level one) with respect to finite subgroups [12]. Whether there is a deeper connection remains to be seen.

\section{Conclusions}

Once again it is apparent how the ideas of supersymmetry can be applied to better understand, and partially unravel a problem that does not necessarily involve supersymmetry at the outset. Here I have shown how one can easily derive the modular properties, of affine $G$-characters, and in addition find methods for generating non-trivial modular invariants. The most interesting of these methods reduces the problem to one of constructing certain Lorentzian, self-dual lattices, and can be thought of as twisting (or more precisely, shifting) the theory by operators of integral spin. The price one appears to pay for the simplicity of this 
lattice method is that the corresponding modular invariants are not good partition functions for conformal theories. However, in practice, it is relatively easy to circumvent this difficulty. First, for a given affine $\mathscr{G}$ at a given level, there are only finitely many Lorentzian self-dual lattices, containing $\sqrt{k+g}(\Gamma(\mathscr{G}), \Gamma(\mathscr{G}))$ as a sub-lattice, or equivalently there are only finitely many integral spin primary fields that can be used to generate shift vectors. It is computationally very easy to generate all the associated modular invariants, and then check to see which combinations of them yield good partition functions. One can also proceed more systematically. Suppose one has applied some shift vector, $\sigma$, of the form (3.15), and obtained an invariant, $I$. By changing the sign of the $I$, if necessary, one obtains an invariant that contains a term of the form $a\left(\chi_{\Lambda_{+}} \overline{\left(\chi_{\Lambda_{-}}\right)}+\chi_{\Lambda_{-}} \overline{\left(\chi_{\Lambda_{+}}\right)}\right)$, where $a$ is a positive constant. If $I$ contains other terms with negative coefficients one can attempt to remove them by either (i) adding on some other known invariant to cancel them or (ii) modifying the Lorentzian lattice by trying to shift with another vector that preserves the desired term, but projects out the offending terms or (iii) manufacturing a new modular invariant by using a shift that generates some or all of the offending terms and then subtracting this new invariant from the old one. Finally, if one obtains an invariant with positive coefficients one, of course, must also make sure that there is a unique ground state.

While it is essential to impose these requirements on the modular invariants of $\hat{\mathscr{G}}$ in order to obtain a conformal field theory based on $\hat{\mathscr{G}}$, it is by no means clear as to whether one has to be quite so stringent if one is interested in coset models based on $\hat{\mathscr{G}}$. That is, one might be able to get an acceptable conformal theory on $G / H$ by using invariants for $\hat{\mathscr{G}}$ and $\hat{\mathscr{H}}$ that would not correspond to conformal theories on $\hat{\mathscr{G}}$ and $\hat{\mathscr{H}}$ separately.

Finally, the classification modular invariants of $G$ can be accomplished by classifying the modular invariants of any subgroup $H$ whose rank is equal to that of $G$. In particular, if one takes $H$ to be the torus of $G$, then all the modular invariants of $\mathscr{G}$ can be obtained from all the modular invariants of bosons compacted on a given lattice. Whether this observation helps with the general classification problem remains to be seen, but it is evident that one can readily generate many of the modular invariants of affine $G$ by using elementary lattice technology.

\section{References}

1. Kazama, Y., Suzuki, H,: Nucl. Phys. B 321, 232 (1989)

2. Lerche, W., Vafa, C., Warner, N.P.: Nucl. Phys. B 324, 427 (1989)

3. Nahm, W.: Duke Math. J. 54, 579 (1987)

Davis, U.C.: Preprints (1988)

Bouwknegt, P., Nahm, W.: Phys. Lett. 188 B, 349 (1987)

4. Walton, M.A.: Nucl. Phys. B 322, 775 (1989)

5. Schellekens, A.N., Warner, N.P.: Nucl. Phys. B 308, 397 (1988)

Lüst, D., Theisen, S.: Preprint MPI-PAE/PTh 83/87

Lerche, W., Schellekens, A.N., Warner, N.P.: Phys. Rep. 177, 1 (1989)

6. Kac, V.G.: Infinite dimensional Lie algebras, second ed. Cambridge: Cambridge University Press 1985 
7. Gepner, D., Witten, E.: Nucl. Phys. B 278, 493 (1986)

8. Goddard, P., Olive, D.: Nucl. Phys. B 257, 266 (1985) Goddard, P., Kent, A., Olive, D.: Phys. Lett. 152 B, 88 (1985)

9. Capelli, A., Itzykson, C., Zuber, J.B.: Nucl. Phys. B 280, 445, (1987); Commun. Math. Phys. 113, 1 (1987)

10. Englert, F., Neveu, A.: Phys. Lett. 163 B, 349 (1985)

11. Vafa, C.: Nucl. Phys. B 273, 592 (1986)

12. Ginsparg, P.: Nucl. Phys. B 295, 153 (1988)

Communicated by A. Jaffe

Received November 9, 1989 
